\title{
MicroRNA-194 restrains the cell progression of non-small cell lung cancer by targeting human nuclear distribution protein $\mathbf{C}$
}

\author{
LIRONG ZHOU, QINGGUO DI, BAOHUA SUN, XIAOSHENG WANG, MIN LI and JIAN SHI \\ Respiratory Department, Cangzhou Central Hospital, Cangzhou, Hebei 061001, P.R. China
}

Received November 4, 2015; Accepted March 7, 2016

DOI: $10.3892 /$ or.2016.4708

\begin{abstract}
NSCLC accounts for over $80 \%$ of all lung cancers and is associated with poor prognosis. Human nuclear distribution C (hNUDC) was predicted to be the target gene of microRNA-194 (miR-194). The present study was designed to demonstrate the mechanism of miR-194 in the regulation of non-small cell lung cancer (NSCLC) via targeting the hNUDC. The hNUDC expression was found to strongly be increased while the miR-194 decreased significantly in the NSCLC cell lines when compared with the healthy controls. Moreover, the luciferase report confirmed the targeting reaction between miR-194 and hNUDC. After transfection with miR-194 mimic into NSCLC cells, we found that the miR-194 overexpression resulted in abnormal nuclear division, decreased cell proliferation and inhibited the expression of hNUDC and $\mathrm{Mpl} / \mathrm{ERK}$ pathway proteins. Furthermore, the hNUDC overexpression affected the suppression effect of miR-194 in 95D cells, indicating that miR-194 suppresses tumor cell process by inhibiting the hNUDC expression. In brief, the present study suggests that the upregulation of miR-194 affects the hNUDC expression, leading to a downregulated expression of $\mathrm{Mpl} / \mathrm{ERK}$ pathway proteins, and suppresses the mitosis and proliferation of NSCLC cells. These results offer a potential therapeutic strategy for the treatment of lung cancer.
\end{abstract}

\section{Introduction}

Lung cancer is one of the most common malignancies globally, with 1.6 million new cases being diagnosed annually and is also the leading cause of cancer deaths worldwide (1).

Correspondence to: Dr Qingguo Di, Respiratory Department, Cangzhou Central Hospital, 16 Xinhuaxi Road, Yunhe, Cangzhou, Hebei 061001, P.R. China

E-mail: diqingguocz@tom.com

Abbreviations: hNUDC, human nuclear distribution C; miR-194, microRNA-194; Mpl, thrombopoietin receptor; ERK1/2, extracellular signal-regulated protein kinases-1 and -2; 3'-UTR, 3'-untranslated region; miRNAs, microRNAs

Key words: non-small cell lung cancer, human nuclear distribution C, microRNA-194, Mpl/ERK pathway
Non-small cell lung cancer (NSCLC) accounts for more than $80 \%$ of all lung cancers accompanied by poor prognosis (2). Despite recent treatment advances, immunotherapy in particular, offers promising treatment alternatives that could help fight disease mortality with minimal impact on normal tissues (3), the chances of survival of NSCLC remain bleak, and novel therapeutic approaches are required.

The human homolog of filamentous fungus Aspergillus nidulans NUDC (nuclear distribution C), called hNUDC, is structurally based on the similarity of its C-terminus to that of the fungal NUDC from Aspergillus nidulans forms (4). NUDC is necessary for nuclei movement following mitosis as well as normal colony growth, which is highly conserved structurally and functionally throughout most evolution (5). The hNUDC has been reported to be involved in mitosis, cytokinesis (6), neuronal migration (7) and hematopoietic cell growth in humans (5). However, the hNUDC function in NSCLC cells has not yet been investigated.

Thrombopoietin receptor $(\mathrm{Mpl})$ is a class I cytokine receptor, belonging to the hematopoietic super family of receptors. hNUDC is confirmed to be the second natural ligand for $\mathrm{Mpl}$ (after thrombopoietin) and binds to the extracellular domain of the $\mathrm{Mpl}$ (8), thus, inducing a sustained activation of the extracellular signal-regulated protein kinases-1 and -2 (ERK1/2), resulting in megakaryocytic proliferation and differentiation (9). The extracellular signal regulated kinase (ERK1/2), also called the mitogen-activated protein kinase (MAPK), reportedly promotes cell survival and chemotherapeutic resistance in NSCLC cell lines (10). ERK signaling pathway was suggested to play a role in the hNUDC overexpression-induced apoptosis (11). Other reports recorded that nuclear and cytoplasmic ERK1/2 activation positively correlated with advanced and aggressive NSCLC tumors (12). These reports suggested that hNUDC may affect the ERK1/2 pathway and regulate the processing of NSCLC cells.

miRNAs have been identified as classical oncogenes or tumor suppressor genes $(13,14)$. In lung cancer, miR-let-7c (15), miR-506 (16) and miR-34a (17) have been identified as tumor suppressors, whereas miR-21 (18), miR-155 (19) and miR-31 (20) were found to be carcinogenesis promoters. Mature miR-194 is involved in both pri-miR-194-2/192 and pri-miR-194-1/215 clusters (14), and has been suggested to be a putative tumor suppressor in liver (21) and ovarian tissues (22). The miR-194 overexpression in these cancer cells suppresses cell migration, invasion and metastasis. The overexpression of 
the miR-194 in lung cancer cell lines has also been reported to suppress metastasis of lung cancer cells (14).

This study helped to identify miR-194 action in the context of non-small cell lung cancer. We first found hNUDC overexpression in NSCLC cell lines and NSCLC patients when compared to healthy controls. Besides, miR-194 was predicted to target hNUDC, which regulated the ERK1/2 pathway. Taken together, our results suggest that miR-194 may provide novel insight into the process of NSCLC via targeting hNUDC.

\section{Materials and methods}

Patients. Twenty-six patients (12 males and 14 females) with non-small cell lung cancer and paired non-tumor lung tissues were consecutively included in this study. Both tumor and non-tumor samples were confirmed by the pathological examinations. Patients were recruited from the Respiratory Department of Cangzhou Central Hospital. The study was approved by the ethics committee of our institution. Informed consent was signed by the participants.

Cell culture. First, 95C and 95D cells were subcloned from the PLA-801 human giant-cell lung carcinoma cell line, but they had different metastatic potentials (23). Human nonsmall cell lung cancer cells HCC827, A549, NCI-H460 and human lung fibroblast (NHLF) cell lines obtained from the American Type Culture Collection (ATCC; Manassas, VA, USA), were cultivated in modified Eagle's medium (MEM; Invitrogen, Carlsbad, CA, USA), supplemented with $20 \%$ fetal bovine serum (FBS; Invitrogen) and $1 \%$ antibiotic-antimycotic (Invitrogen), while the NSCLC cell lines 95C and 95D were grown in MEM supplemented with $10 \%$ FBS and $1 \%$ antibiotic-antimycotic. All the cells were incubated at $37^{\circ} \mathrm{C}$ in a humidified $21 \% \mathrm{O}_{2}, 5 \% \mathrm{CO}_{2}$ atmosphere.

$q R T-P C R$. The total RNA was extracted from TRIzol reagent (Invitrogen) following the manufacturer's instructions. For miR-194 detection, reverse transcription was performed using One Step PrimeScript miRNA cDNA Synthesis kit (Takara, Dalian, China), following the primers: 5'-UGUAACAGCA ACUCCAUGUGGA-3' (sense); common antisense primer, 5'-GACTGTTCCTCTCTTCCTC-3'. For mRNA detection of hNUDC, the cDNA was generated using M-MLV reverse transcriptase (Clontech Laboratories, Palo Alto, CA, USA), and amplified following the primers: 5'-AGACCTGCCCAA TTACCGC-3' (sense); 5'-GCTCCCCATCAATGATCGCT-3' (antisense). To analyze the gene expression, the qRT-PCR mixture system containing the cDNA templates, primers and SYBR-Green qPCR Master Mix were subjected to qRT-PCR quantification according to the standard methods. $\beta$-actin and U6 SnRNA were used as the internal control of the mRNA or miRNA, respectively. The human $\beta$-actin primers: 5'-GAT CATGTTTGAGACCTTC-3' (sense); 5'-GGCATACCCCTCG TAGATG-3' (antisense), the U6 primers: 5'-GCTTCGGCAG CACATATACTAAAAT-3' (sense); 5'-CGCTTCACGAATTT GCGTGTCAT-3' (antisense). Relative gene expression was quantified by $2^{-\Delta \Delta \mathrm{Ct}}$ method.

Western blot analysis. A total of $25 \mu \mathrm{g}$ proteins were loaded and separated via sodium dodecyl sulfate-polyacrylamide gel electrophoresis and then electrotransferred to nitrocellulose membranes (Amersham, Little Chalfont, UK). The membranes were then blocked in $2.5 \%$ non-fat milk for $1 \mathrm{~h}$ at $37^{\circ} \mathrm{C}$. After washing with Tris-buffered saline with Tween, the membranes were incubated with primary antibodies against Mpl, ERK1/2, c-myc, $\beta$-actin (Santa Cruz Biotechnology, Santa Cruz, CA, USA) and p-CRBE (Cell Signaling Technology, Danvers, MA, USA) at $4{ }^{\circ} \mathrm{C}$ overnight. Then, the peroxidase-conjugated secondary antibody (Wuhan Boster Biological Technology, Ltd., Wuhan, China) diluted in 1:1,000 was added and incubated for $1 \mathrm{~h}$ at room temperature. The immunoreactive protein bands were then visualized using an enhanced chemiluminescence detection system (Amersham).

Dual-luciferase reporter assay. The target gene was predicted by TargetScan (http://www.targetscan.org/). The 3'-UTR fragment of hNUDC mRNA containing the target sequence (CUGUUAC) of miR-194 was amplified by RT-PCR. The fragment, designated hNUDC 3'-UTR, was inserted into the pMIR-REPORT ${ }^{\mathrm{TM}}$ luciferase reporter vector $(M l u \mathrm{I}$ and HindIII restriction enzyme sites; Ambion, Austin, TX, USA). Another expressing vector was also constructed by the insertion of a mutated hNUDC 3'-UTR in which the target sequence of miR-194 was mutated into CUGUAAC using the QuickChange Site-Directed Mutagenesis kit (Stratagene, Santa Clara, CA, USA). The recombinant reporter vectors with normal and mutated hNUDC 3'-UTR were co-transfected with miR-194 into 95D cells using the TransMessenger ${ }^{\mathrm{TM}}$ Transfection reagent (Qiagen, Hilden, Germany). The luciferase assay was performed according to the manufacturer's protocol. The relative luciferase activities were normalized to that of the control cells.

Transfection assay. miRNA mimics and inhibitors, specific for miR-194 (Invitrogen), were used to increase and silence miR-194 expression in 95C and 95D cell lines, respectively. Two hundred pmoles of miR-194 mimics, miR-194 mimic control, miR-194 inhibitor, and inhibitor control (Ambion) were transfected into $3 \times 10^{6} 95 \mathrm{C}$ and $95 \mathrm{D}$ cells for $48 \mathrm{~h}$ by electroporation using a Nucleofector instrument, respectively. After transfection, the cells were allowed to recover by incubating them for $4 \mathrm{~h}$ at $37^{\circ} \mathrm{C}$. The experiment was replicated thrice for data calculations.

Giemsa staining. For Giemsa staining, incubation of the $1 \times 10^{4}$ 95C and 95D cells was done on $35 \mathrm{~mm}$ of the cell petri dishes with a coverslip in each dish. After $48 \mathrm{~h}$, the coverslips were immobilized using $100 \%$ methyl alcohol and then air dried. The cells were then treated with Giemsa staining solution and the cellular morphology was microscopically observed (Leica AF6000; Leica Microsystems, Wetzlar, Germany).

MTT assay. Cell viability was assessed using 3-(4,5-dimethylthiazol-2-yl)-2,5-diphenyltetrazolium bromide (MTT) assay. Shortly afterwards, cells were transfected according to the above description and were seeded in 96-well plates at $6 \times 10^{3}$ cells/well. The surviving fractions were determined at $0,24,48,72,96$ and $120 \mathrm{~h}$. Thereafter, the old medium was discarded and fresh medium containing MTT $(5 \mathrm{mg} / \mathrm{ml}$ MTT in PBS; Shanghai Sangon Biological Engineering Technology, 

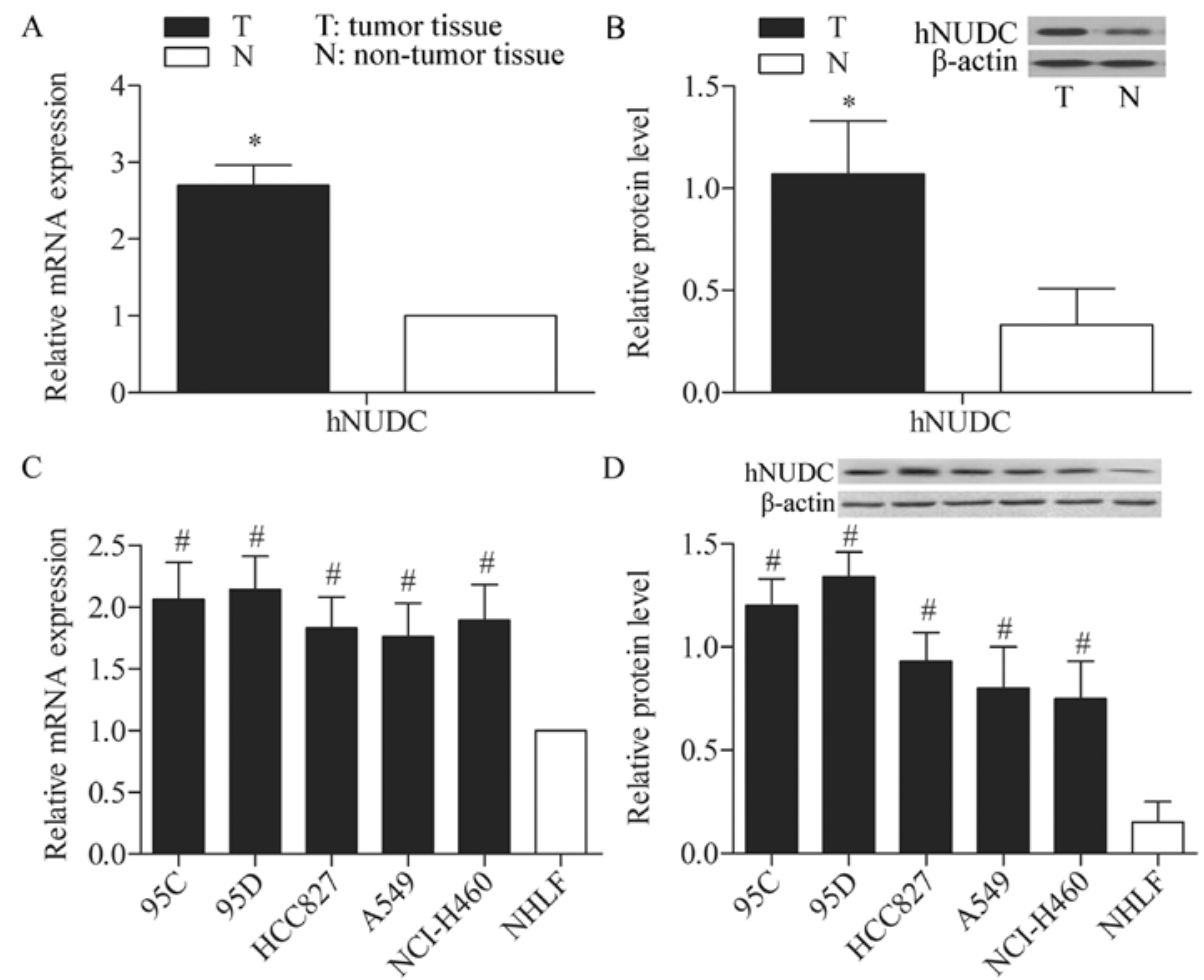

Figure 1. Inverse level of hNUDC in NSCLC and adjacent non-tumor lung tissues. The inverse level was also detected in the NSCLC cells (95C, 95D, HCC827, A549, NCI-H460) and NHLF cells. (A) The mRNA expression levels of hNUDC in the NSCLC and adjacent non-tumor lung tissues were measured by quantitative real-time PCR (qRT-PCR); (B) the protein expression levels of hNUDC in NSCLC and adjacent non-tumor lung tissues were measured by western blot analysis; (C) the mRNA expression of hNUDC in NSCLC cells (95C, 95D, HCC827, A549 and NCI-H460) and NHLF cells was assessed by qRT-PCR assays; (D) western blotting assay was used to confirm the hNUDC expression profile in the cell lines mentioned above. The relative protein expression was quantified using Image-Pro Plus 6.0 software and normalized to $\beta$-actin; data are presented as the mean $\pm \mathrm{SD}$ of three experiments. ${ }^{*} \mathrm{P}<0.05$ vs. normal cases, ${ }^{\#} \mathrm{P}<0.05$ vs. the NHLF cell lines.

Shanghai, China) was added and incubated for an additional $4 \mathrm{~h}$. Then, cell viability was measured with a spectrophotometer (Bio-Rad Laboratories, Hercules, CA, USA) at $470 \mathrm{~nm}$. Each experiment was performed in triplicate.

Cell cycle analysis. Cell cycle analysis was determined by flow cytometry (BD Biosciences, San Jose, CA, USA). In short, 95C and 95D cells at $1 \times 10^{6}$ cells/well were cultured in 6-well plates and transfected with $50 \mathrm{nM}$ of the miR-194 mimics, miR-194 inhibitor or their respective control RNA for $48 \mathrm{~h}$. The cells were then harvested and fixed in $70 \%$ ice-cold ethanol for $24 \mathrm{~h}$, followed by staining with propidium iodide (PI). The different cell cycle phases were analyzed with the FACSCalibur instrument using CellQuest software (Becton-Dickinson, Mountain View, CA, USA).

hNUDC overexpression. The hNUDC overexpression was achieved by PCR amplification using hNUDC cDNA as a template, and the hNUDC expressing vector was constructed by inserting the hNUDC cDNA into pcDNA 3.1 vector. The recombinant plasmid and other agents were co-transfected into $3 \times 10^{6} 95 \mathrm{D}$ cells using a Nucleofector instrument. Fortyeight hours later, subsequent experiments were performed on the cells. The experiment was replicated thrice for data calculations.

NSCLC xenografts. Nine NOD/SCID mice (Jackson Laboratory, Bar Harbor, ME, USA) (male; body weight,
20-22 g; age, 8 weeks old) were purchased from the Institute of Zoology, Chinese Academy of Medical Sciences. 95D cells were transfected with miR-194 or negative control miRNA (NC pre-miR ${ }^{\mathrm{TM}}$; Ambion) following same transfection conditions. Cells $\left(5 \times 10^{6}\right)$ 95D transfected with miR-194 were injected subcutaneously into the right flank of NOD/SCID mice $(n=9)$. Cells transfected with negative control miRNA were injected into the left flank of NOD/SCID mice $(n=9)$. The tumor volumes were measured daily after the injection, and all the rats were assigned to euthanasia at the end of measurements (on day 27). All animal experiments were performed in accordance with current prescribed guidelines and under a protocol approved by the Institutional Animal Care and Use Committee.

Statistical analysis. All results are presented as mean \pm SD. Statistical analysis was carried out using one-way analysis of variance (ANOVA) followed by Bonferroni test. The difference was considered statistically significant at $\mathrm{P}<0.05$.

\section{Results}

Inverse level of hNUDC in NSCLC and adjacent non-tumor lung tissues. The level of hNUDC expression was detected in NSCLC and adjacent non-tumor lung tissues via qPCR and western blot analysis. The results indicated that the level of hNUDC expression was significantly higher in tumor tissues compared with matched non-tumor tissues $(\mathrm{P}<0.05)($ Fig. 1A 
A

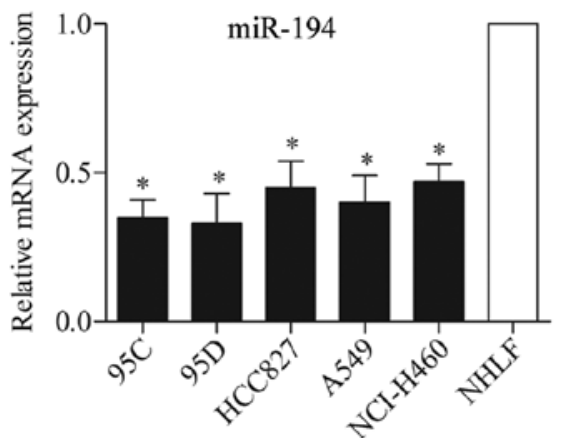

$\mathrm{C}$

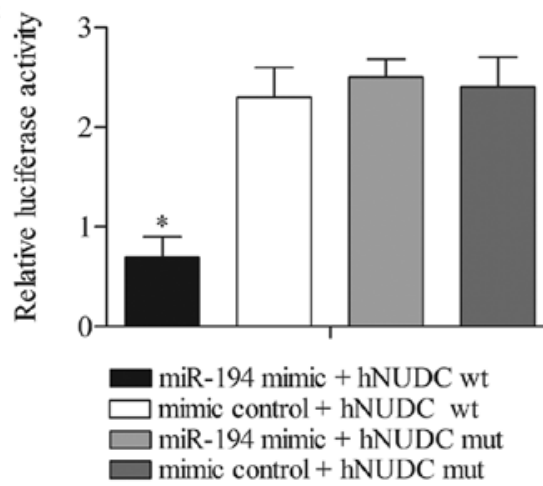

B

\author{
AGGUGUACCUCAACGACAAUGU Hsa-miR-194 \\ | | | | ||| |
}

UCCCCAGUUGGCCUA-CUGUUACA NUDC3'UTR (186-193)

UCCCCAGUUGGCCUA-CUGUAACA Mutation $(\boldsymbol{\Delta})$

$\boldsymbol{\Delta}$

D

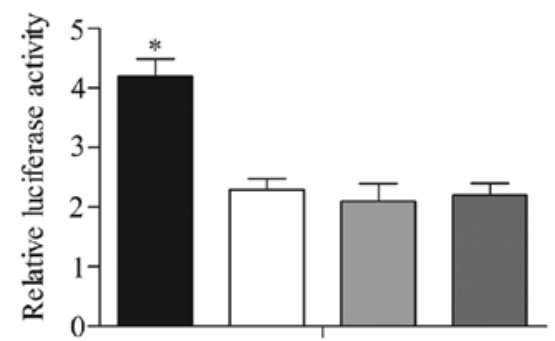

miR-194 inhibitor + hNUDC wt

Inhibitor control + hNUDC wt

miR-194 inhibitor + hNUDC mut

Inhibitor control + hNUDC mut

Figure 2. The target relationship between miR-194 and hNUDC. The target gene was predicted by TargetScan database and identified by luciferase activity report. (A) The level of miR-194 expression was detected in 95C, 95D, HCC827, A549 and NCI-H460 and NHLF cell lines by qPCR; (B) the wild and the mutant hNUDC 3'-UTR contained the target sequence of miR-194 was predicted by TartgetScan; (C) the 95D cells were transfected with miR-194 mimic or mimic control luciferase reporter vectors containing the wild-type (wt) or mutant (mut) hNUDC 3'-UTR; (E) a similar luciferase assay was performed in 95D cells treated with the miR-194 inhibitor or control. Luciferase activity was demonstrated as firefly luciferase normalized to Renilla luciferase. Data are presented as the mean $\pm \mathrm{SD}$ of 3 experiments. ${ }^{*} \mathrm{P}<0.05$ vs. the control group.

and B). The level of hNUDC expression was also detected in 95C, 95D, HCC827, A549, NCI-H460 and NHLF cell lines by qPCR. The results indicated that the mRNA expression level of the hNUDC was significantly higher in the NSCLC cells (95C, 95D, HCC827, A549 and NCI-H460) compared with NHLF cells $(\mathrm{P}<0.05)$ (Fig. 1C). The levels of the hNUDC protein expression in the three cell lines examined by western blot analysis were consistent with the mRNA expression levels (Fig. 1D). The results suggested that the levels of hNUDC expression were significantly elevated in the NSCLC patients and NSCLC cells compared with normal control.

hNUDC is targeted by miR-194. Assuming that the hNUDC level was increased in the NSCLC cell lines (especially in 95D), in order to obtain the biological role of hNUDC in the NSCLC cells, it was of interest to identify the microRNA that could target hNUDC. Predicted by the bioinformatic software programs TargetScan, we found that hNUDC was targeted by miR-194. The level of miR-194 expression was detected in 95C, 95D, HCC827, A549, NCI-H460 and NHLF cell lines by qPCR. The results indicated that the mRNA level of miR-194 expression was significantly lower in NSCLC cells (95C, 95D, HCC827, A549 and NCI-H460) compared with NHLF cells $(\mathrm{P}<0.05)$ (Fig. 2A). The potential binding target sites of miR-194 were found in the 3'-UTR of hNUDC gene (Fig. 2B). To experimentally confirm that hNUDC was an authentic target of miR-194 in the 95D cells, the plasmid pMIR-REPORThNUDC-wt or pMIR-REPORT-hNUDC-mut was transfected into $95 \mathrm{D}$ cells together with miR-194 mimics or mimic control. After $48 \mathrm{~h}$ of transfection, the results showed that the luciferase activity in the hNUDC-wt with miR-194 mimics transfection group was significantly reduced compared with the other three groups (Fig. 2C). Consistently, the luciferase reporter vectors of the hNUDC-wt and hNUDC-mut were co-transfected with the miR-194 inhibitors or inhibitor controls into 95D cells. The results showed that the luciferase activity in the hNUDC-wt with miR-194 inhibitor co-transfection group was increased significantly compared with the other three groups (Fig. 2D). The data mentioned above demonstrated that hNUDC is a genuine target of miR-194.

miR-194 causes abnormal mitosis of NSCLC cells via targeting $h N U D C$. As hNUDC is associated with human nuclear migration, we examined whether the overexpression or inhibition of miR-194 was capable of affecting cell mitosis by targeting hNUDC. miR-194 mimic was used to amplify the miR-194 expression, whereas a synthetic inhibitor specific for miR-194 was employed to suppress the expression of endogenous miR-194 in NSCLC cell lines. The efficiency of this miR-194 mimic or inhibitor was confirmed by qPCR assay (Fig. 3A and B), and 95C and 95D cells were transfected with miR-194 mimic, mimic control, miR-194 inhibitor and inhibitor control, separately, while the mRNA and protein level of hNUDC expression were examined by qRT-PCR (Fig. 3C and D) and western blot analysis (Fig. 3E and F), respectively. The quantified relative protein expression is summarized in 
A

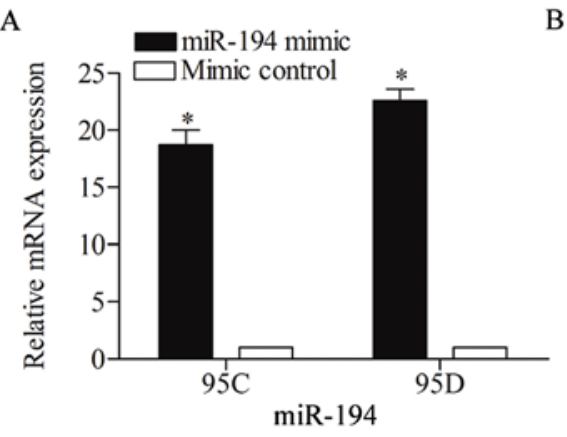

D

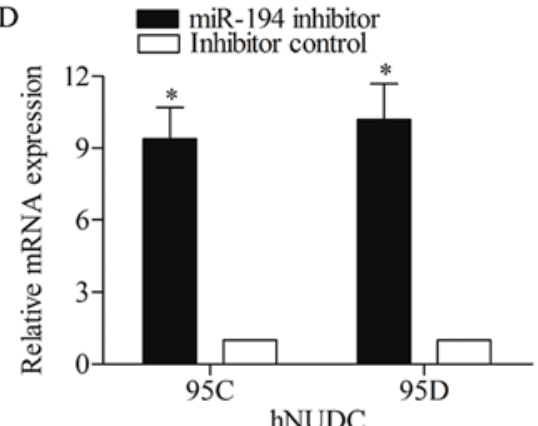

G

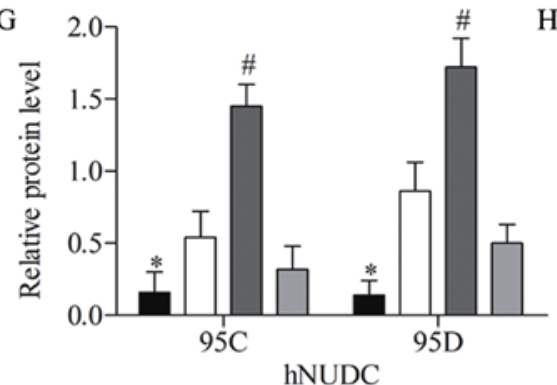

hNUDC
B

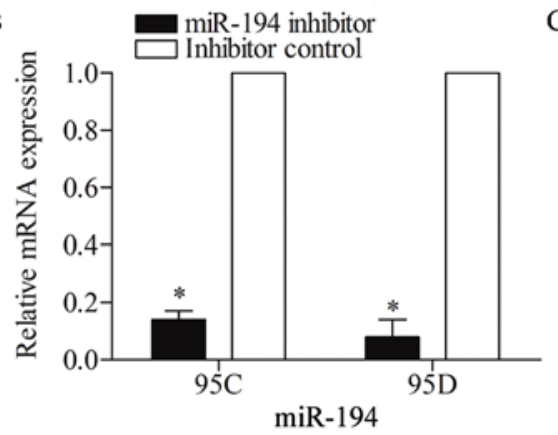

E
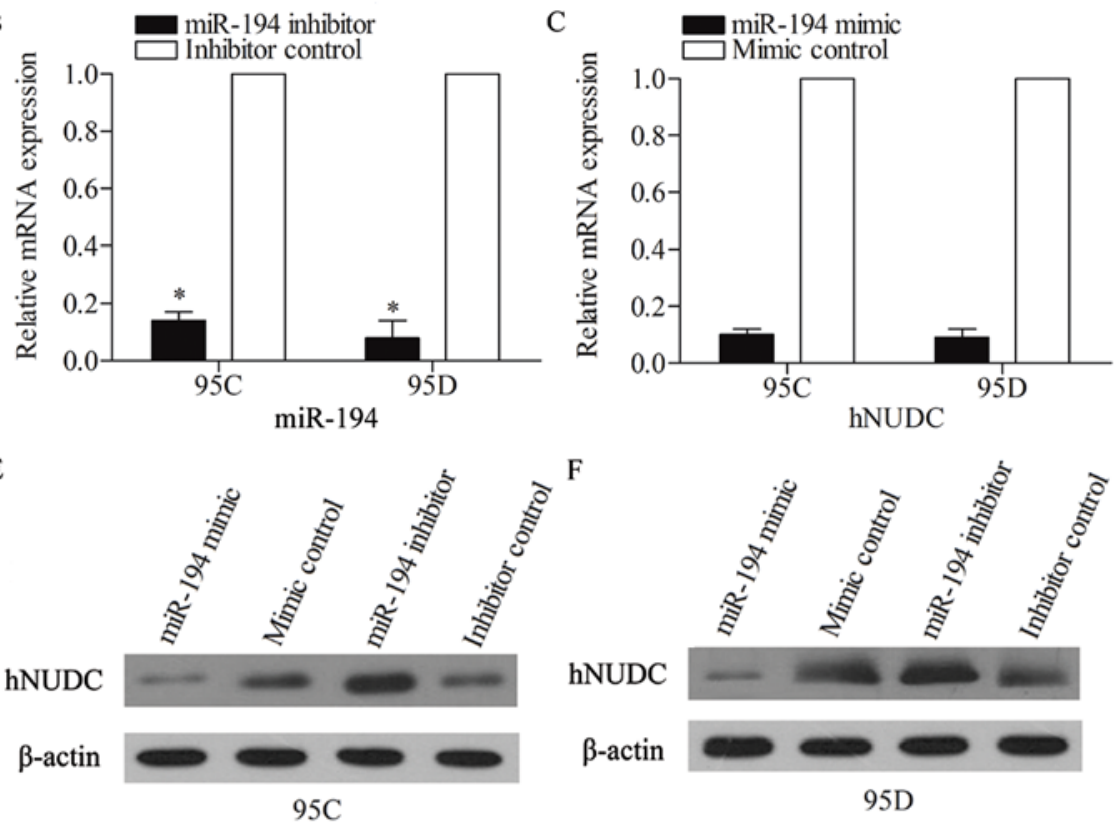

$F$

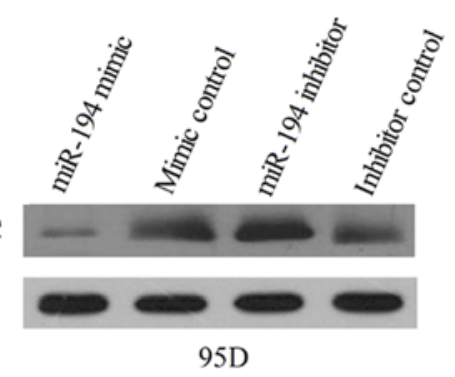

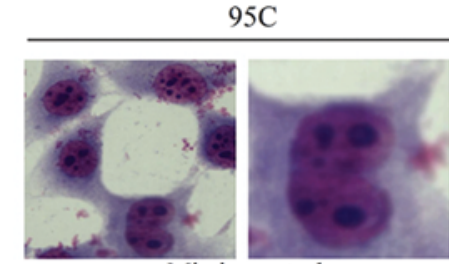

Mimic control

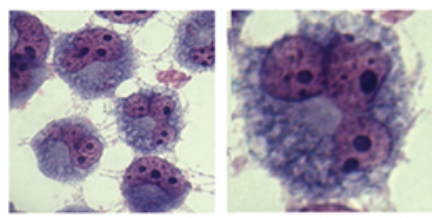

miR-194 mimic

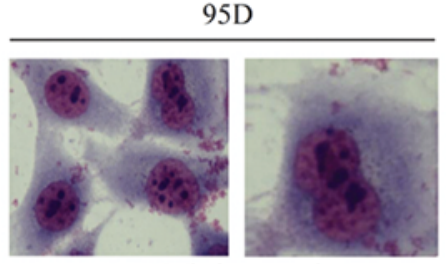

Mimic control

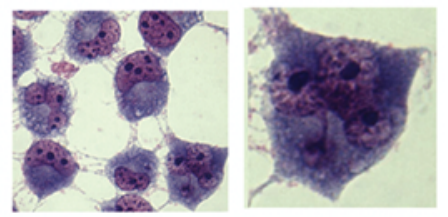

miR-194 mimic

Figure 3. miR-194 induces abnormal mitosis of NSCLC cells via targeting hNUDC. 95C and 95D cells were transfected with miR-194 mimic, mimic control, miR-194 inhibitor or inhibitor control, respectively. (A) The expression levels of miR-194 in 95C and 95D cells co-transfected with miR-194 mimic or mimic control were measured by qPCR; (B) the levels of miR-194 expression in 95C and 95D cells co-transfected with miR-194 inhibitor or inhibitor control were measured by qPCR; (C) the relative mRNA levels of hNUDC in 95C and 95D cells co-transfected with miR-194 mimic or mimic control were measured by qRT-PCR; (D) the mRNA levels of hNUDC in 95C and 95D cells co-transfected with miR-194 inhibitor or inhibitor control were measured by qRT-PCR; western blot analysis was performed to measure the relative protein levels of hNUDC in 95C cells (E) and 95D cells (F); (G) the relative protein expression was quantified using Image-Pro Plus 6.0 software and normalized to $\beta$-actin; $(\mathrm{H})$ the cell nuclear division of 95C and 95D cells was detected by Giemsa staining assay. The data are presented as the mean $\pm \mathrm{SD}$ of three experiments. ${ }^{*} \mathrm{P}<0.05$ vs. the mimic control group; ${ }^{*} \mathrm{P}<0.05$ vs. the inhibitor control group.

Fig. 3G. The results showed that both the mRNA and protein levels of hNUDC were significantly downregulated when treated with miR-194 mimic, and increased when treated with miR-194 inhibitor compared with control groups. The Giemsa staining assay showed the nucleus was divided into two in the mimic control group in 95C and 95D cells. However, the nucleus exhibited abnormal division due to the overexpression of miR-194, three nuclei were detected within one cell in 95C cells, and sometimes even four (Fig. 3H). These results indicated that miR-194 affected the NSCLC cell mitosis via regulating the expression of hNUDC.

The effect of miR-194 on the cell cycle and proliferation of 95C and $95 \mathrm{D}$ cells. To further investigate the impact of miR-194 on the NSCLC cells, we studied whether the miR-194 overexpres- sion was capable of affecting cell cycle and proliferation. The 95C and 95D cells were transfected with miR-194 mimic and mimic control separately. The analysis of the cell cycle indicated that miR-194 overexpression induced an accumulation of 95C cells in G0/G1 phase (Fig. 4A), and an accumulation of $95 \mathrm{D}$ cells in $\mathrm{G} 2 / \mathrm{M}$ phase compared with mimic control (Fig. 4B), implying a cell cycle arrest in 95C and 95D cells with the change in the miR-194 levels. The cell cycle distribution in each group in 95C and 95D cells is summarized in Fig. 4C and $\mathrm{D}$. The cell proliferation assay was performed in the cell lines, and miR-194 mimic was observed to strongly suppress the $95 \mathrm{C}$ cell growth compared with mimic control group (Fig. 4E). Similar MTT results were obtained in 95D cells, in which the cell proliferation rate was decreased under the treatment of miR-194 mimic (Fig. 4F) compared with mimic 
A

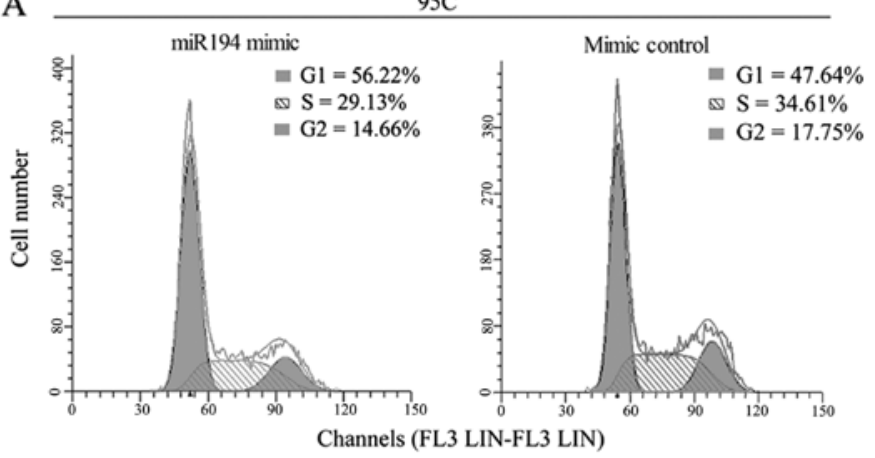

B

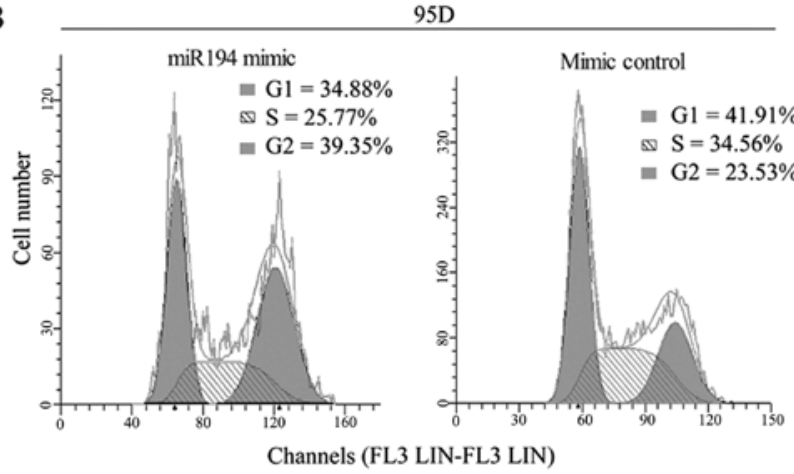

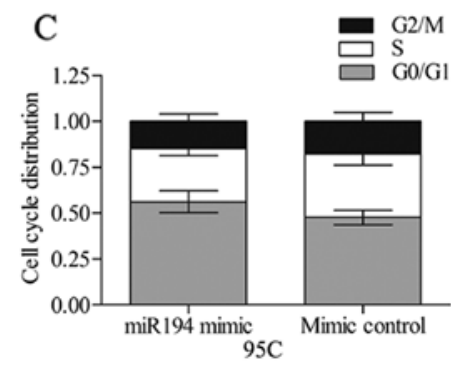
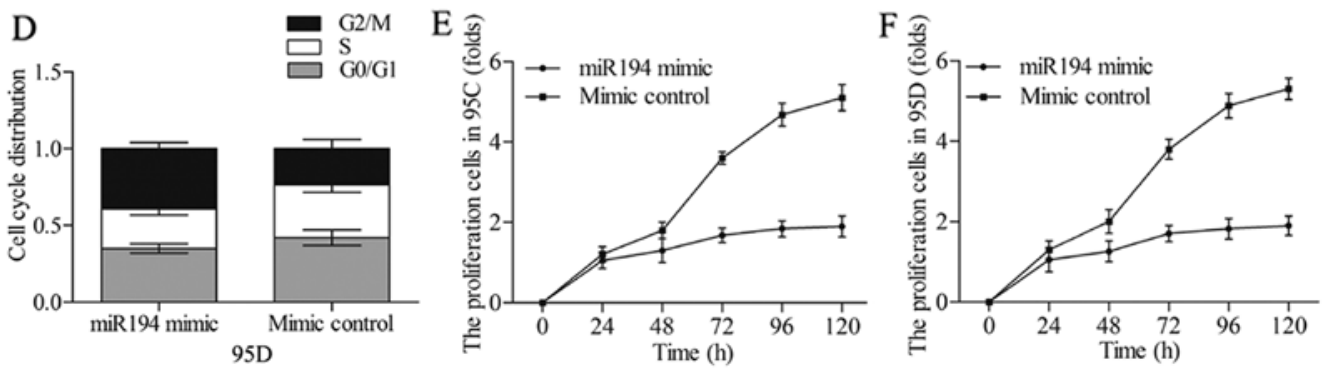

Figure 4. The effect of miR-194 on the cell cycle and proliferation of 95C and 95D cells. 95C and 95D cells were transfected with miR-194 mimic and mimic control, respectively. The cell cycle analysis of 95C (A) and 95D cells (B) with the miR-194 mimic or with mimic control were determined by flow cytometric analysis; the distributions of G0/G1, S and G2/M phases were evaluated in 95C (C) and 95D (D) cells transfected with miR-194 or with mimic control for $48 \mathrm{~h}$. The proliferation of $95 \mathrm{C}(\mathrm{E})$ and $95 \mathrm{D}(\mathrm{F})$ cells was determined at the time-points indicated by the MTT assays. All the experiments were repeated three times with three replicates.
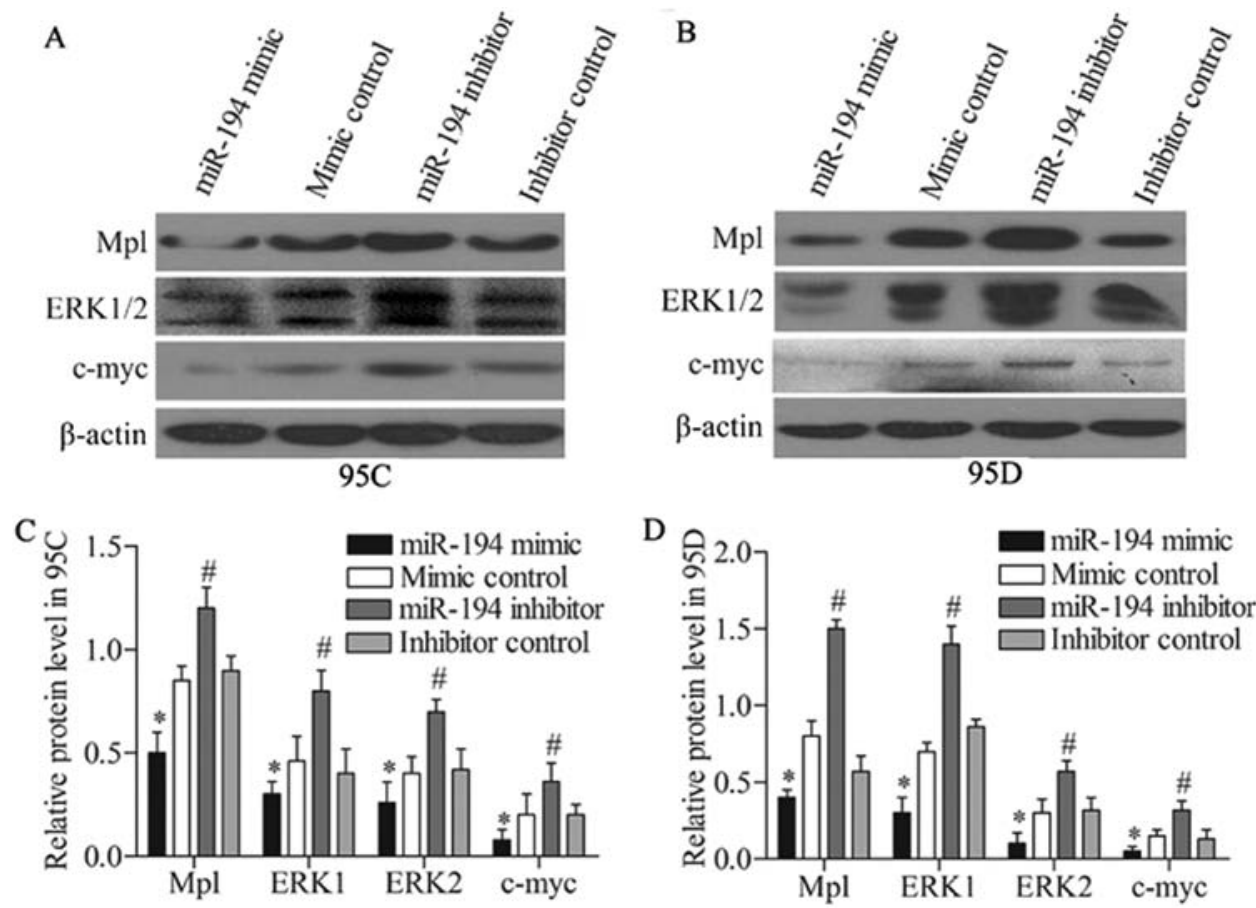

Figure 5. miR-194 regulates Mpl/ERK pathway via targeting hNUDC. The 95C and 95D cells were transfected with the miR-194 mimic, mimic control and miR-194 inhibitor or inhibitor control, separately. (A) The protein expression levels of Mpl/ERK pathway in 95C cells were measured by western blotting; (B) a similar western blotting assay was performed in 95D cells; (C) the relative protein expressions in 95C cells were quantified using Image-Pro Plus 6.0 software and normalized to $\beta$-actin; (D) relative protein expressions in $95 \mathrm{D}$ cells. Data are presented as the mean $\pm \mathrm{SD}$ of all three experiments. "P<0.05 vs. mimic control, ${ }^{\#} \mathrm{P}<0.05$ vs. inhibitor control.

control. These findings suggest that miR-194 suppresses the cell cycle by decelerating the G0/G1 phase in $95 \mathrm{C}$ cells and the $\mathrm{G} 2 / \mathrm{M}$ phase in $95 \mathrm{D}$ cells, and inhibits cell proliferation in the NSCLC cells. 
A
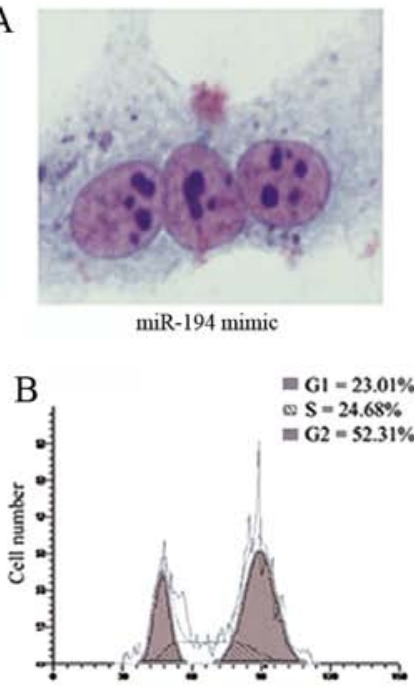

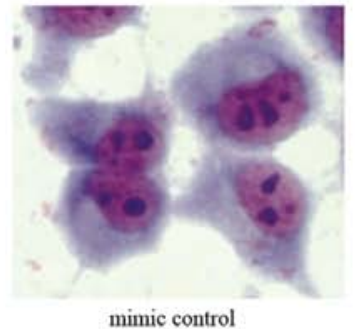

mimic control

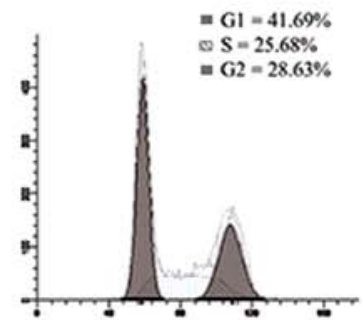

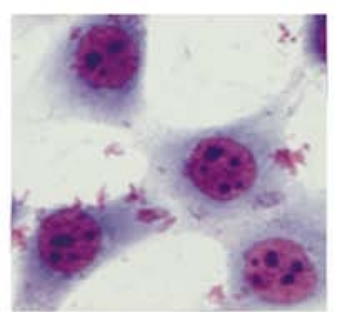

hNUDC overexpression

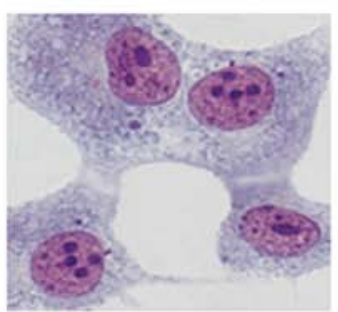

miR-194 mimic + overexp hNUDC

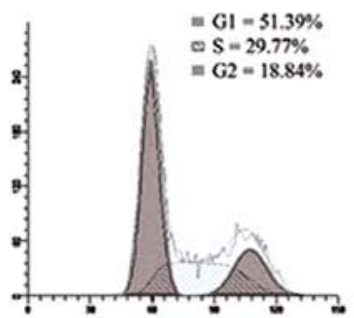

$=\mathrm{G} 1=38.4 \%$

es $\mathrm{S}=25.16 \%$

$=\mathrm{G} 2=36,43$

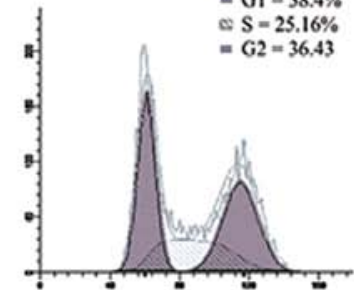

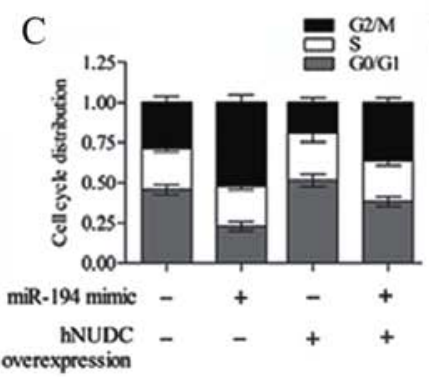
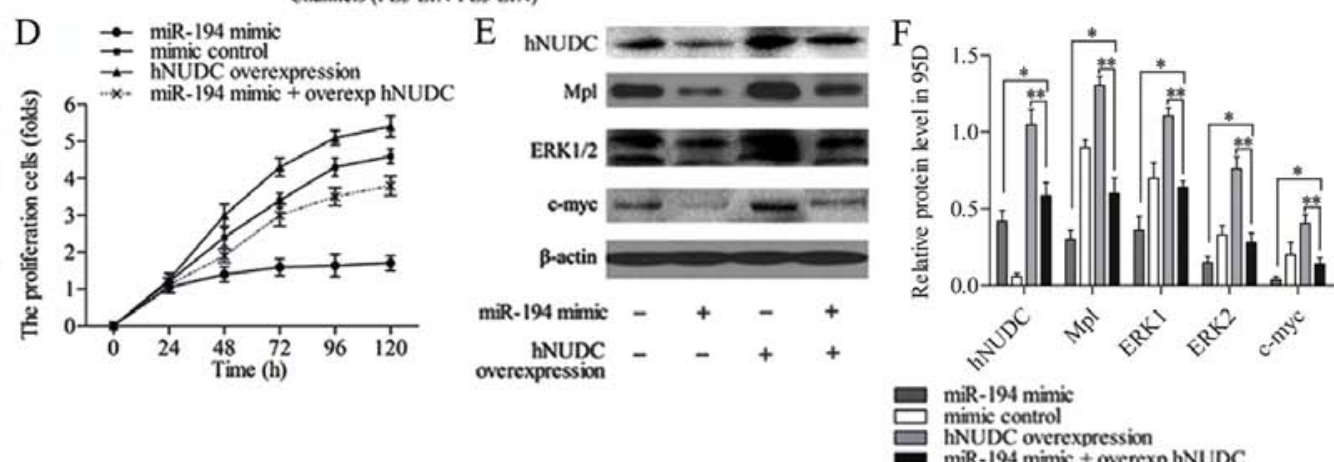

Figure 6. hNUDC restoration is necessary to affect the miR-194 suppression on the NSCLC cells and ERK pathway. Cells were treated with miR-194 mimic or mimic control with or without the overexpression hNUDC vector. (A) The cell nuclear division of 95D cells in each group was detected by Giemsa staining assay; (B) the cell cycle progression was conducted by flow cytometric analysis; (C) the distributions of the G0/G1, S and G2/M phases were evaluated in 95D cells; (D) MTT assay was employed to examine the proliferation rates of 95D cells treated with miR-194 mimicor mimic control with or without the overexpession hNUDC vector; (E) the relative hNUDC, Mpl, ERK1/2 and c-myc expressions were detected via western blotting; (F) the relative protein expressions in $95 \mathrm{D}$ cells were quantified using Image-Pro Plus 6.0 software and normalized to $\beta$-actin. The data are shown as means \pm SD of three independent experiments. ${ }^{*} \mathrm{P}<0.05,{ }^{* *} \mathrm{P}<0.01$.

miR-194 inhibits the Mpl/ERK pathwaybytargeting hNUDC. hNUDC has been reported to promote cell proliferation and differentiation via activation of the thrombopoietin receptor $(\mathrm{Mpl})$ and the extracellular signal-regulated protein kinases-1 and -2 (ERK1/2) pathway (9). Thus, we detected the effect of miR-194 overexpression and the suppression on the expression of Mpl/ERK pathway, which included $\mathrm{Mpl}$, ERK1/2 and c-myc genes. The protein expression was detected via western blot analysis. The results revealed that miR-194 mimic effectively decreased the expressions of Mpl, ERK1/2 and c-mycin 95C cells compared with mimic control. Whereas, miR-194 inhibitor promoted the expression of these genes compared with mimic control (Fig. 5A). Western blotting assay was also performed in 95D cells, miR-194 mimic intensively restrained the expressions of $\mathrm{Mpl}, \mathrm{ERK} 1 / 2$ and c-myc compared with the mimic control group. Stronger expression of these genes was detected when they were subjected to the miR-194 inhibitor treatment compared with inhibitor control (Fig. 5B). The quantified relative protein expressions of $\mathrm{Mpl} / \mathrm{ERK}$ pathway in $95 \mathrm{C}$ and $95 \mathrm{D}$ cells is summarized in Fig. 5C and D. The data mentioned above suggested that miR-194 restrained the Mpl/ ERK pathway in the NSCLC cells.

miR-194 suppression of hNUDC is necessary to influence NSCLC cells and ERK pathway. Given that hNUDC is the target of miR-194, it was of interest to study whether the hNUDC mediated the effect of miR-194 on the process of NSCLC cells and Mpl/ERK pathway. To determine whether the overexpression of hNUDC counteracted the effect of miR-194 in 95D cells, miR-194 mimic or mimic control with or without the hNUDC overexpression vector were co-transfected into 95D cells. Giemsa staining assay showed the presence of three nuclei within one cell in the miR-194 mimic group, however, cell division was found to be normal after hNUDC was overexpressed. This was because hNUDC is related to nucleus distribution, and the hNUDC overexpression resulted in an increase in migration ability of the nucleus. Cells co-transfected with miR-20b mimic plus overexpression hNUDC vector exhibited relatively abnormal mitosis compared with overexpression hNUDC group (Fig. 6A). Flow cytometric analysis of the cell cycle progression demonstrated 


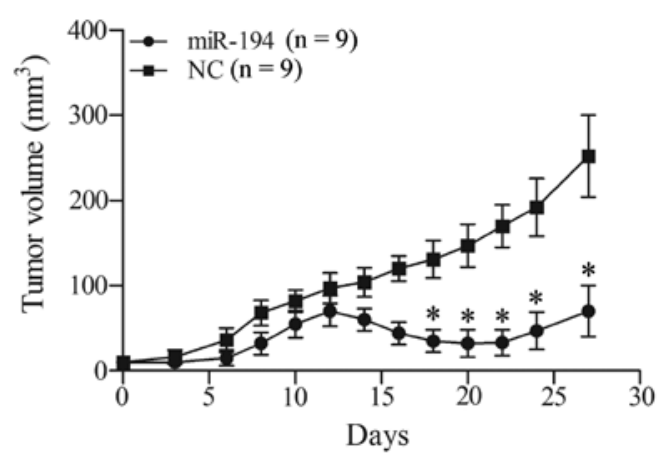

Figure 7. miR-194 suppresses tumor growth of lung cancer xenografts. 95D cells were transfected with miR-194 or negative control miRNA (NC) ex vivo and injected subcutaneously into $9 \mathrm{NOD} / \mathrm{SCID}$ mice using $5 \times 10^{6}$ cells/flank (day 0). 95D cells transfected with negative control miRNA (NC) were injected into the opposite flank of the same animals that also received miR-194 treated cells. Measurements of tumor volumes were taken daily till day 27. Standard deviations are indicated in the graph. ${ }^{*} \mathrm{P}<0.05$.

that the $\mathrm{G} 2 / \mathrm{M}$ arrest in $95 \mathrm{D}$ cells could be obviously detected in the miR-194 mimic group. After co-transfecting the miR-194 mimic and overexpression of hNUDC in 95D cells, the G2/M phase was extended compared with overexpression hNUDC group (Fig. 6B). The cell cycle distribution in each group in 95D cells is summarized in Fig. 6C. The results indicated that the inhibition effect of the cell cycle by miR-194 mimic could be reversed by the hNUDC overexpression.

To further confirm the enhanced expression of hNUDC counteracting the effect of miR-194 mimic in 95D cells, MTT assay indicated that the increasing hNUDC and miR-194 levels could suppress the proliferation rates of 95D cells treated with overexpression of hNUDC alone (Fig. 6D). Western blotting was used to measure the expression of hNUDC, Mpl, ERK1/2 and c-myc (Fig. 6E), and the brands were quantified in Fig. 6F. In the miR-194 mimic plus overexpression hNUDC group, the protein expression of hNUDC, Mpl, ERK1/2 and c-myc was strongly decreased compared with the overexpression hNUDC group $(\mathrm{P}<0.01)$, and increased when compared with the miR-194 mimic group $(\mathrm{P}<0.05)$. The results illustrated that the hNUDC overexpression was able to offset the effect of miR-194 inhibition on Mpl/ERK pathway.

miR-194 reduces tumor growth in xenograft models of lung cancer. To further explore the tumor suppressor effect of miR-195, we assessed tumor growth of xenografts that were transfected with miR-194 or negative control miRNA was subcutaneous injected into NOD/SCID mice. As shown in Fig. 7, miR-194 inhibited tumor growth of the 95D xenograft. At early time-points, tumors that developed from miR-194treated cells were smaller compared with their control tumors. At later time-points, tumor growth was resumed but still significantly reduced compared with the control group. The results in vivo demonstrated the inhibition effect of miR-194 acting towards cell growth of NSCLC.

\section{Discussion}

NSCLC is the leading cause of cancer death. The hNUDC overexpression has been reported to induce differentiation in megakaryocytes (11). In the present study, the hNUDC expres- sion is significantly increased in the NSCLC tumor tissues and NSCLC cell lines compared with the matched non-tumor tissues and NHLF cells. To investigate the role of hNUDC in NSCLC, the non-coding region, 3'-UTR of hNUDC was studied. miRNAs are non-coding RNAs that can suppress the expression of the protein-coding genes by binding to the target sequence at the 3'-UTR region of the target gene (24). In the present study, we predicted miR-194 is the targeted miRNA for the hNUDC, which may be involved in the NSCLC pathogenesis.

The strikingly decreased level of miR-194 expression has been reported in many cancer cell lines. In gastric cancer (GC), miR-194 was significantly downregulated and RING box protein1 (RBX1) was upregulated in GC tissues, the upregulation of miR-194 can inhibit proliferation and invasion of GC cells, possibly by targeting RBX1 (25). Moreover, the overexpression of miR-194 regulates osteoblast differentiation through modulating STAT1-mediated Runx2 nuclear translocation (26). Additionally, miR-194 expression was also found to be in a strongly negative association with metastasis in the clinical specimens of NSCLC, as it suppressed the metastasis of NSCLC through regulation of the expression of BMP1 and p27kip1 (14). In the present study, the level of hNUDC was strongly increased in NSCLC cells compared with NHLF cells, and the target connection between hNUDC and miR-194 was predicted by bioinformatic software program TargetScan. Therefore, we chose miR-194 to be the targeted miRNA for hNUDC. The results showed that miR-194 decreased significantly in the NSCLC cells compared with the healthy controls, which was consistent with a prior study by $\mathrm{Wu}$ et al (14). To verify the targeting reaction between miR-194 and hNUDC, luciferase reporter vectors of the wild-type and mutant hNUDC were constructed. The results confirmed that hNUDC is a target gene for miR-194.

Nuclear migration is essential for growth, development, and cellular function of eukaryotes (27). hNUDC protein plays an important role in nuclear migration (28). The increased hNUDC expression was found to be closely associated with cell malignant hyperplasia in nasopharyngeal carcinoma. A report indicated that anti-NUDC antibody could inhibit the growth of nasopharyngeal carcinoma cell lines (27). From the perspective mentioned above, targeting hNUDC with certain small RNAs offers a novel strategy to prevent the abnormal growth of the NSCLC cells. Therefore, to investigate the role of miR-194 in NSCLC cell process via targeting hNUDC, we detected the effect of miR-194 overexpression on the mitosis, cell proliferation and cell cycle ability of 95C and 95D cells. The results showed that the miR-194 mimic strongly inhibited the hNUDC expression, inducing abnormal nuclear division and decreased cell proliferation rate. Multiple evidence confirms that $95 \mathrm{C}$ and $95 \mathrm{D}$ cells have relatively low and high metastasis potential $(23,29,30)$. Consistent with these studies, the relatively low level of malignancy of $95 \mathrm{C}$ cells renders them more sensitive to the external factors compared with 95D cells. The cell growth was withheld in G1 phase during the treatment of miR-194 mimic compared with mimic control. However, as 95D cells were characterized by rapid rate of DNA synthesis and cell growth, they became accumulated in G2 phase in the treatment with miR-194 mimic. It is reported that cells arrested in G2/M achieved nuclear enlargement and 
polyplodization, possibly through failure to complete both mitosis and cytokinesis (31). These results confirmed that miR-194 acted as a negative control in the cell processing in NSCLC cells via targeting hNUDC.

hNUDC has been found to act as a secondary ligand for $\mathrm{Mpl}$ involved in regulating proliferation and differentiation of different types of megakaryocytes $(32,33)$. hNUDC plays a key role in the megakaryocytes undergoing endomitosis through the interaction with $\mathrm{Mpl}$ (34). Native hNUDC and Mpl were seen around the nuclei and in cytoplasm extensions at all stages of megakaryocytic development (8). A report also indicated that the overexpression of hNUDC activated the EKR1/2 pathway (11), and this activation was profound and prolonged in an Mpl-dependent manner (34). The present study revealed that the miR-194 mimic inhibited the expressions of $\mathrm{Mpl} /$ ERK/c-myc pathway proteins by a decrease in hNUDC expression. The downregulation of c-myc is an important event that has been connected to the terminal differentiation and growth arrest of several cell types (35). In order to further confirm the inhibitory effect of miR-194 on the expression of Mpl/ERK pathway proteins and on the cell process of NSCLC cells via targeting hNUDC. hNUDC was overexpressed in 95D cells and the results revealed that the overexpression of hNUDC restored the inhibitory effect of the miR-194 on the mitosis, cell growth and protein expression of Mpl/ERK pathway in 95D cells. These results suggest that miR-194 is a newly identified miRNA that suppresses the expression of hNUDC, at least in NSCLC cells (possibly in other types of human cells). This finding can contribute to a clearer understanding of the regulatory network of $\mathrm{Mpl} / \mathrm{ERK}$ pathway in human cancers. hNUDC is revealed as a new NSCLC-associated tumorpromoting gene in this study. Notably, this could facilitate the development of a therapeutic strategy targeting hNUDC for lung cancer treatment in studies in the future.

It has been reported that the overexpression of miR-194 suppressed invasion and migration of liver cancer cells in mice (36), indicating the tumor-suppressing role of miR-194 in vivo. In this study, the overexpression of miR-194 inhibited tumor growth of the 95D xenograft in NOD/SCID mice at early time-points, however, miR-194 delayed the onset of $95 \mathrm{D}$ tumor growth and did not reduce growth of the resulting tumors from day 21 to 27 . We speculate that miR-194 induces a growth delay of the transplanted cells that is overcome with time. Multiple administrations or stable expression systems might be necessary to suppress tumor growth more efficiently.

In conclusion, our results demonstrate that the miR-194 overexpression affects the hNUDC expression, resulting in abnormal nuclear division, suppresses cell growth by cell cycle arrest in G1 or G2 phase and downregulates Mpl/ERK pathway proteins in NSCLC cells. The present study is an important step towards understanding the pathogenesis of NSCLC and implicates miR-194 as a potential therapeutic target for NSCLC.

\section{Acknowledgements}

The authors would like to thank the members of the Respiratory Department, Cangzhou Central Hospital, for providing helpful discussions concerning the present study.

\section{References}

1. Jemal A, Bray F, Center MM, Ferlay J, Ward E and Forman D: Global cancer statistics. CA Cancer J Clin 61: 69-90, 2011.

2. Jemal A, Siegel R, Ward E, Hao Y, Xu J, Murray T and Thun MJ: Cancer statistics, 2008. CA Cancer J Clin 58: 71-96, 2008.

3. Marschner JP, Quaratino S and Forssmann U: Non-small cell lung cancer, NSCLC. In: Cancer Immunotherapy Meets Oncology. Springer, pp193-201, 2014.

4. Miller BA, Zhang M-Y, Gocke CD, De Souza C, Osmani AH, Lynch C, Davies J, Bell L and Osmani SA: A homolog of the fungal nuclear migration gene nudC is involved in normal and malignant human hematopoiesis. Exp Hematol 27: 742-750, 1999.

5. Gocke CD, Reaman GH, Stine C, Zhang MY, Osmani SA and Miller BA: The nuclear migration gene NudC and human hematopoiesis. Leuk Lymphoma 39: 447-454, 2000.

6. Aumais JP, Williams SN, Luo W, Nishino M, Caldwell KA, Caldwell GA, Lin SH and Yu-Lee LY: Role for NudC, a dyneinassociated nuclear movement protein, in mitosis and cytokinesis. J Cell Sci 116: 1991-2003, 2003.

7. Aumais JP, Tunstead JR, McNeil RS, Schaar BT, McConnell SK, Lin SH, Clark GD and Yu-Lee LY: NudC associates with Lis1 and the dynein motor at the leading pole of neurons. J Neurosci 21: RC187, 2001.

8. Pan RM, Yang Y, Wei MX, Yu XB, Ge YC and Xu P: A microtubule associated protein (hNUDC) binds to the extracellular domain of thrombopoietin receptor $(\mathrm{Mpl})$. J Cell Biochem 96: 741-750, 2005.

9. Tang YS, Zhang YP and Xu P: hNUDC promotes the cell proliferation and differentiation in a leukemic cell line via activation of the thrombopoietin receptor (Mpl). Leukemia 22: 1018-1025, 2008.

10. Brognard J and Dennis PA: Variable apoptotic response of NSCLC cells to inhibition of the MEK/ERK pathway by small molecules or dominant negative mutants. Cell Death Differ 9: 893-904, 2002.

11. Xiao Y, Zheng Y, Tan P, Xu P and Zhang Q: Overexpression of nuclear distribution protein (hNUDC) causes pro-apoptosis and differentiation in Dami megakaryocytes. Cell Prolif 46: 576-585, 2013.

12. Vicent S, López-Picazo JM, Toledo G, Lozano MD, Torre W, Garcia-Corchón C, Quero C, Soria JC, Martín-Algarra S, Manzano RG, et al: ERK1/2 is activated in non-small-cell lung cancer and associated with advanced tumours. Br J Cancer 90: 1047-1052, 2004

13. Yang CH, Pfeffer SR, Sims M, Yue J, Wang Y, Linga VG, Paulus E, Davidoff AM and Pfeffer LM: The oncogenic microRNA-21 inhibits the tumor suppressive activity of FBXO11 to promote tumorigenesis. J Biol Chem 290: 6037-6046, 2015.

14. Wu X, Liu T, Fang O, Leach LJ, Hu X and Luo Z: miR-194 suppresses metastasis of non-small cell lung cancer through regulating expression of BMP1 and $\mathrm{p} 27^{\mathrm{kip} 1}$. Oncogene 33: $1506-1514,2014$

15. Zhao B, Han H, Chen J, Zhang Z, Li S, Fang F, Zheng Q, Ma Y, Zhang J, Wu N, et al: MicroRNA let-7c inhibits migration and invasion of human non-small cell lung cancer by targeting ITGB3 and MAP4K3. Cancer Lett 342: 43-51, 2014.

16. Yin M, Ren X, Zhang X, Luo Y, Wang G, Huang K, Feng S, Bao X, Huang K, He X, et al: Selective killing of lung cancer cells by miRNA-506 molecule through inhibiting NF- $\mathrm{BB}$ p65 to evoke reactive oxygen species generation and p53 activation. Oncogene 34: 691-703, 2015.

17. Shi Y, Liu C, Liu X, Tang DG and Wang J: The microRNA miR-34a inhibits non-small cell lung cancer (NSCLC) growth and the CD44hi stem-like NSCLC cells. PLoS One 9: e90022, 2014.

18. Ma Y, Xia H, Liu Y and Li M: Silencing miR-21 sensitizes non-small cell lung cancer A549 cells to ionizing radiation through inhibition of PI3K/Akt. BioMed Res Int 2014: Article ID 617868,2014

19. Gao F, Chang J, Wang H and Zhang G: Potential diagnostic value of miR-155 in serum from lung adenocarcinoma patients. Oncol Rep 31: 351-357, 2014

20. Dong Z, Zhong Z, Yang L, Wang S and Gong Z: MicroRNA-31 inhibits cisplatin-induced apoptosis in non-small cell lung cancer cells by regulating the drug transporter ABCB9. Cancer Lett 343: 249-257, 2014. 
21. Bao C, Li Y, Huan L, Zhang Y, Zhao F, Wang Q, Liang L, Ding J, Liu L, Chen $\mathrm{T}$, et al: NF- $\mathrm{BB}$ signaling relieves negative regulation by miR-194 in hepatocellular carcinoma by suppressing the transcription factor HNF-1 $\alpha$. Sci Signal 8: ra75-ra75, 2015.

22. Nakamura K, Sawada K, Kinose Y, Hashimoto K, Mabuchi S and Kimura T: Identification of microRNA which regulates paclitaxel resistance of ovarian cancer cells - a potential of miR-194 by attenuating paclitaxel resistance through the down-regulation of oncogene BMI-1. Cancer Res 74 (19 Suppl): 4386, 2014.

23. Lu YL: Spontaneous metastasis of clonal cell subpopulations of human lung giant cell carcinoma after subcutaneous inoculation in nude mice. Zhonghua Zhong Liu Za Zhi 11: 1-7, 1989 (In Chinese).

24. Ambros V, Lee RC, Lavanway A, Williams PT and Jewell D: MicroRNAs and other tiny endogenous RNAs in C. elegans. Curr Biol 13: 807-818, 2003

25. Chen X, Wang Y, Zang W, Du Y, Li M and Zhao G: miR-194 targets RBX1 gene to modulate proliferation and migration of gastric cancer cells. Tumour Biol 36: 2393-2401, 2015.

26. Li J, He X, Wei W and Zhou X: MicroRNA-194 promotes osteoblast differentiation via downregulating STAT1. Biochem Biophys Res Commun 460: 482-488, 2015.

27. Chen Y, Li T, Qu S and Tang X: Expression and effects of nuclear distribution $C$ (NUDC) protein in nasopharyngeal carcinoma cell lines. Ai Zheng 25: 708-712, 2006 (In Chinese).

28. Zhang MY, Huang NN, Clawson GA, Osmani SA, Pan W, Xin P, Razzaque MS and Miller BA: Involvement of the fungal nuclear migration gene nudC human homolog in cell proliferation and mitotic spindle formation. Exp Cell Res 273: 73-84, 2002.

29. Hou M, Tan L, Wang X and Zhu YS: Antisense Tiam1 downregulates the invasiveness of $95 \mathrm{D}$ cells in vitro. Acta Biochim Biophys Sin (Shanghai) 36: 537-540, 2004.
30. Ren T, Wen ZK, Liu ZM, Liang YJ, Guo ZL and Xu L: Functional expression of TLR9 is associated to the metastatic potential of human lung cancer cell: Functional active role of TLR9 on tumor metastasis. Cancer Biol Ther 6: 1704-1709, 2007.

31. Lin S-H, Nishino M, Luo W, Aumais JP, Galfione M, Kuang J and Yu-Lee LY: Inhibition of prostate tumor growth by overexpression of NudC, a microtubule motor-associated protein. Oncogene 23: 2499-2506, 2004.

32. Pang SF, Li XK, Zhang Q, Yang F and Xu P: Interference RNA (RNAi)-based silencing of endogenous thrombopoietin receptor $(\mathrm{Mpl})$ in Dami cells resulted in decreased hNUDC-mediated megakaryocyte proliferation and differentiation. Exp Cell Res 315: 3563-3573, 2009

33. Wei MX, Yang Y, Ge YC and Xu P: Functional characterization of hNUDC as a novel accumulator that specifically acts on in vitro megakaryocytopoiesis and in vivo platelet production. J Cell Biochem 98: 429-439, 2006.

34. Zhang YP, Tang YS, Chen XS and Xu P: Regulation of cell differentiation by hNUDC via a Mpl-dependent mechanism in NIH 3T3 cells. Exp Cell Res 313: 3210-3221, 2007.

35. Gartel AL and Shchors K: Mechanisms of c-myc-mediated transcriptional repression of growth arrest genes. Exp Cell Res 283: 17-21, 2003

36. Meng Z, Fu X, Chen X, Zeng S, Tian Y, Jove R, Xu R and Huang W: miR-194 is a marker of hepatic epithelial cells and suppresses metastasis of liver cancer cells in mice. Hepatology 52: $2148-2157,2010$ 